\title{
High molecular weight hyaluronan for treatment of chronic shoulder pain associated with glenohumeral arthritis
}

This article was published in the following Dove Press journal:

Medical Devices: Evidence and Research

25 July $201 \mathrm{I}$

Number of times this article has been viewed

\author{
Arnold J Weil \\ Non-Surgical Orthopedics \\ PC, Marietta, GA, USA
}

Correspondence: Arnold J Weil Non-Surgical Orthopedics PC, 335 Roselane Street, Marietta, GA 30060, USA

Tel +l 7704204645

Fax +I 7702345526

Email nonsurgicalmd@lowbackpain.com
Background: There is insufficient evidence to determine whether intra-articular injections may be effective for treatment of glenohumeral osteoarthritis. Euflexxa ${ }^{\circledR}$ (high molecular weight hyaluronate), a bioengineered high molecular weight hyaluronan, has been shown to be a safe and effective treatment for patients with knee osteoarthritis. There is also support for the use of hyaluronate injection for the treatment of chronic shoulder pain associated with osteoarthritis or rotator cuff damage. This small-scale exploratory study was conducted to evaluate the safety and efficacy of high molecular weight hyaluronate for the treatment of chronic shoulder pain associated with osteoarthritis.

Methods: Subjects with glenohumeral osteoarthritis and chronic pain $(n=27)$ received one injection per week for 3 weeks of high molecular weight hyaluronate and were assessed for changes in pain (100 mm visual analog scale [VAS]), range of motion, and the subject's and physician's global assessment over 26 weeks. Subjects were also assessed for pain, stiffness, and physical functioning using the Western Ontario and McMaster Universities Arthritis Index (WOMAC). Finally, responses were evaluated using modified Outcome Measures in Rheumatoid Arthritis Clinical Trials (OMERACT)-Osteoarthritis Research Society International (OARSI) Proposition D criteria. Safety was assessed by recording adverse events.

Results: High molecular weight hyaluronate significantly improved pain (VAS, WOMAC), range of motion, stiffness, and physical functioning scores; $77.8 \%$ of subjects were rated as having an OMERACT-OARSI Proposition D high response. There were no serious adverse events, and none were considered to be related to treatment.

Conclusion: Treatment with high molecular weight hyaluronate improves pain, stiffness, and range of motion, and may have an acceptable safety and tolerability profile. A randomized, double-blind, placebo-controlled clinical trial may be warranted to investigate further the efficacy and safety of intra-articular high molecular weight hyaluronate for treatment of chronic shoulder pain in patients with glenohumeral osteoarthritis.

Keywords: Euflexxa ${ }^{\circledR}$, hyaluronan, osteoarthritis, pain, range of motion, shoulder

\section{Introduction}

Persistent shoulder pain, regardless of the definition used, is a common disorder. A survey of 312 general practice patients in the United Kingdom indicated that $31 \%-48 \%$ had shoulder pain depending on the definition used, and $27 \%$ of all respondents had shoulder pain regardless of the definition. ${ }^{1}$ Results summarized in a comprehensive review of shoulder disorders indicated that the annual incidence of this condition is about $7 \%$, the 1 -year prevalence is $51 \%$, and the lifetime prevalence is approximately $10 \% .^{2}$ 
Chronic shoulder pain can result from bursitis, tendinitis, rotator cuff tear, adhesive capsulitis, impingement syndrome, necrosis, glenohumeral osteoarthritis, and degenerative joint disease. ${ }^{3}$ Rotator cuff disorders, adhesive capsulitis, and glenohumeral osteoarthritis are all common causes. Analysis of 692 office visits for shoulder pain collected through the National Ambulatory Medical Care Survey from 1993 to 2000 indicated that rotator cuff syndrome of the shoulder was the most common diagnosis $(9.6 \%)$, followed by shoulder pain not otherwise specified (8.5\%) and osteoarthritis $(5.6 \%) .{ }^{4}$ Degenerative joint disease of the shoulder is also common. The shoulder is the third most common joint to require surgical reconstruction after the knee and hip. ${ }^{5}$ The prevalence of idiopathic adhesive capsulitis ranges from $0.2 \%$ to $5.0 \%$ and it is most common in elderly patients. ${ }^{6}$

Effective treatment of chronic shoulder pain depends on an accurate diagnosis of the more common etiologies, ie, rotator cuff disorders, adhesive capsulitis, acromioclavicular osteoarthritis, glenohumeral osteoarthritis, and instability. Activity modification and analgesic medications comprise the initial treatment in most cases. If this does not lead to improvement, or if the initial presentation is of sufficient severity, a trial of physical therapy that focuses on the specific diagnosis is indicated. Combined steroid and local anesthetic injections can be used alone or as an adjuvant to physical therapy. ${ }^{7,8}$ There is insufficient evidence to support or refute the use of oral pharmacologic treatments for shoulder arthritis. There is also insufficient evidence to determine whether intra-articular steroid injection is effective for the treatment of glenohumeral osteoarthritis. As few as $20 \%$ of patients with chronic shoulder pain self-report complete recovery after therapeutic intervention, and current recommendations support few nonsurgical options for the treatment of shoulder osteoarthritis. ${ }^{5,9,10}$

There is limited support for the use of hyaluronan injection for the treatment of chronic shoulder pain associated with osteoarthritis or rotator cuff damage. Metaanalysis of results from 19 randomized controlled trials (2120 participants) revealed that intra-articular injection of hyaluronate can decrease symptoms of chronic painful shoulder. This treatment was associated with reductions in pain and improvements in total functional scores. ${ }^{11}$ Results from a study of 30 consecutive patients with idiopathic glenohumeral osteoarthritis who failed to respond to standard conservative measures and then received weekly intra-articular hyaluronate injections over 3 weeks in their affected shoulders indicated that this treatment significantly improved pain at 6-month follow-up. ${ }^{12}$ A study of 660 patients with persistent shoulder pain and limitations resulting from glenohumeral joint osteoarthritis, rotator cuff tear, and/or adhesive capsulitis in whom conventional therapy failed and who received either intra-articular hyaluronate or phosphate-buffered saline solution, was conducted, with patient follow-up for 26 weeks. Patients who received hyaluronate had greater pain relief than control subjects, and this effect was driven by results from patients with osteoarthritis. There was no significant benefit of treatment in patients with either rotator cuff tendonitis or adhesive capsulitis. ${ }^{13}$ Results from 93 elderly patients with rotator cuff tear arthropathy indicated that treatment with hyaluronate significantly decreased symptoms for 4 months. ${ }^{14}$ Intra-articular hyaluronate injection was also shown to decrease pain and improve range of motion and ability to accomplish daily activities in a study of 51 patients with rotator cuff lesions but no tears. ${ }^{15}$

Euflexxa $^{\circledR}(1 \%$ sodium hyaluronate $)$ is a highly purified high molecular weight hyaluronate preparation produced by bioengineering and extracted from bacterial cells. ${ }^{16}$ It has been shown to be safe and effective for treatment of patients with knee osteoarthritis. ${ }^{17,18}$ The purpose of this study was to evaluate the safety and efficacy of high molecular weight hyaluronate using three intra-articular injections for treatment of chronic shoulder pain associated with glenohumeral osteoarthritis.

\section{Methods}

This was a nonrandomized, open-label study conducted in compliance with and according to 21 CRF Parts 50, 54, 56, and 812 . The final protocol was approved by the Sterling local institutional review board. The study, conducted at a single center, aimed to enroll 25 subjects. All subjects were required to be 30-79 years of age, provide written informed consent, have shoulder pain for at least 6 months but less than 5 years, with a pain intensity score $>5$, and be willing to discontinue all pain medications 4 hours before each clinic visit. Twentyseven of 32 subjects recruited for the study met these criteria and had a diagnosis of osteoarthritis confirmed by radiographs taken within the previous 6 months and chronic pain with osteoarthritis as the primary source. Enrolled subjects also had a limited shoulder range of motion in at least one direction for the following active range of motions: internal rotation $60^{\circ}$, external rotation of $80^{\circ}$, or abduction with scapula fixed of $80^{\circ}$, and had an active range of motion of at least $40 \%$ in all directions. The pain medication regimen must have been stable for at least 1 month before treatment and subjects agreed to maintain pain medication doses within $\pm 30 \%$ of the baseline dose during the course of the study.

Subjects were excluded if they had a history of full-thickness rotator cuff tear or joint effusion, a diagnosis 
of chronic acromioclavicular disease, active epicondylitis, or active carpal tunnel disease, had more than two corticosteroid injections in the previous 3 months, or had an infection or inflammatory condition of the trial shoulder. Other grounds for exclusion were acute or inflammatory arthropathy in the trial shoulder, history of systemic inflammatory arthropathies, diagnosis of cancer within 5 years, receipt of any hyaluronate agent or other chondroprotective agents in the trial shoulder, hypersensitivity to hyaluronate products, eggs, birds, or feathers, and history of substance abuse, alcohol abuse, or a psychiatric condition, that, in the opinion of the investigator, could interfere with participation in the study. Subjects were also excluded for any medical condition that might increase the risk associated with intra-articular injections or other concomitant diseases that, in the opinion of the investigator, would interfere with the evaluation of the trial treatment. Individuals involved in litigation involving workers' compensation, who were pregnant, planning to become pregnant or lactating, or who had participated in a clinical trial within the past 4 weeks were also excluded. All subjects received three injections (one each, in weeks 0 [baseline], 1, and 2 of high molecular weight hyaluronate $(2.5 \mathrm{~mL}$ each) using standard injection techniques in the anterior or posterior approach. Subjects were evaluated at screening and baseline, and at weeks $1,2,6,14,26$, and 27 (last evaluation by telephone).

\section{Efficacy}

Efficacy assessments included: pain, measured on a $100 \mathrm{~mm}$ visual analog scale at baseline and weeks 1, 2, 3, 6, 14, and 26; range of motion, measured as a percentage at baseline and weeks 1, 2, 3, 6, 14, and 26; subject's global assessment, measured on a five-point scale (higher score corresponds to worse condition) at weeks 3, 6, 14, and 26; physician's global assessment, measured on a $100 \mathrm{~mm}$ visual analog scale at the same times; internal and external rotation of the shoulder and scapula degrees, measured at baseline and weeks 1, 2, 3, 6, 14, and 26; and Western Ontario and McMaster Universities Arthritis Index (WOMAC) pain, stiffness, and physical functioning measured at baseline and weeks 1, 2, 3, 6, 14, and 26. Responses to treatment were also evaluated using modified Outcome Measures in Rheumatoid Arthritis Clinical Trials (OMERACT)-Osteoarthritis Research Society International (OARSI) Proposition D criteria. A high response was defined as $\geq 50 \%$ or $\geq 20$-point improvement in pain as measured by the $100 \mathrm{~mm}$ visual analog scale, or in range of motion as measured by the 100-point American Shoulder and Elbow Surgeons (ASES) shoulder score of $\geq 50 \%$ and absolute change of $\geq 20 \mathrm{~mm}$. Moderate improvement was defined as having met at least two of the following: pain reduction $\geq 20 \%$ and absolute change $\geq 10$ points; ASES shoulder score $\geq 20 \%$ and a change of $\geq 10$ points on a 100 -point scale; and/or subject global assessment $\geq 20 \%$ and absolute change $\geq 10$ points. Safety was assessed by recording adverse events. Changes from baseline in means values for all efficacy variables were evaluated by means of paired $t$-tests.

\section{Results \\ Subjects}

Of the 32 subjects recruited for the study, five failed the screening visit and were excluded from the analysis. Efficacy and safety analyses were based on the remaining 27 subjects who were enrolled and completed the trial. Baseline demographic and clinical characteristics for these subjects are summarized in Table 1.

Table I Subject demographic and clinical characteristics

\begin{tabular}{|c|c|c|c|c|}
\hline \multicolumn{5}{|l|}{ Demographics $(n=27)$} \\
\hline \multicolumn{5}{|l|}{ Gender (n [\%]) } \\
\hline Male & \multicolumn{4}{|c|}{14 (5I.9) } \\
\hline Female & \multicolumn{4}{|c|}{$13(48.1)$} \\
\hline Age, years (mean [SD]) & \multicolumn{4}{|c|}{$59.1(10.3)$} \\
\hline Clinical characteristics at visit I & $\mathbf{n}$ & Mean (SD) & Minimum & Maximum \\
\hline Pain VAS scores, mm & 27 & $56.37(16.62)$ & 5.0 & 83.0 \\
\hline Total pain assessment & 27 & $6.70(1.17)$ & 5.0 & 9.0 \\
\hline Degree of internal rotation & 27 & $47.04(\mid 5.7 I)$ & 15.0 & 72.0 \\
\hline Degree of external rotation & 27 & $64.96(16.48)$ & 20.0 & 85.0 \\
\hline Scapula degree & 27 & $87.52(19.13)$ & 40.0 & 160.0 \\
\hline Range of motion, $\%$ & 27 & $76.81(12.27)$ & 50.0 & 100.0 \\
\hline WOMAC pain VAS scores, mm & 25 & $14.44(5.45)$ & 3.0 & 23.0 \\
\hline WOMAC stiffness scores & 25 & II.84 (4.06) & 0.0 & 18.0 \\
\hline WOMAC physical functioning scores & 25 & $72.12(25.94)$ & 17.0 & 124.0 \\
\hline
\end{tabular}

Abbreviations: VAS, visual analog score; WOMAC, Western Ontario and McMaster Universities Arthritis Index; SD, standard deviation. 


\section{Efficacy}

Treatment with high molecular weight hyaluronate significantly improved both pain (visual analog scale) and range of motion (\%) over 26 weeks of follow-up (both $P<0.0001$, Figure 1). Treatment with high molecular weight hyaluronate significantly improved (decreased) WOMAC pain scores, WOMAC stiffness scores, and WOMAC physical functioning scores over 26 weeks of follow-up (all $P<0.0001$, Figure 2). Treatment also resulted in a significantly improved range of motion over 26 weeks of follow-up, including internal rotation $(P<0.0001$, Figure $3 \mathrm{~A})$, external rotation $(P<0.0001$, Figure $3 \mathrm{~B})$, and scapular degrees $(P<0.0001$, Figure $3 \mathrm{C})$. Treatment with high molecular weight hyaluronate also significantly improved both subject's and physician's global assessments at 26 weeks after treatment $(P=0.0006$ and $P=0.0011$, respectively; Figure 4).

Based on OMERACT-OARSI Proposition D criteria that combined three outcome measures at the end of the trial, 21 subjects $(77.8 \%)$ were considered to have a high response

A

Time (week)

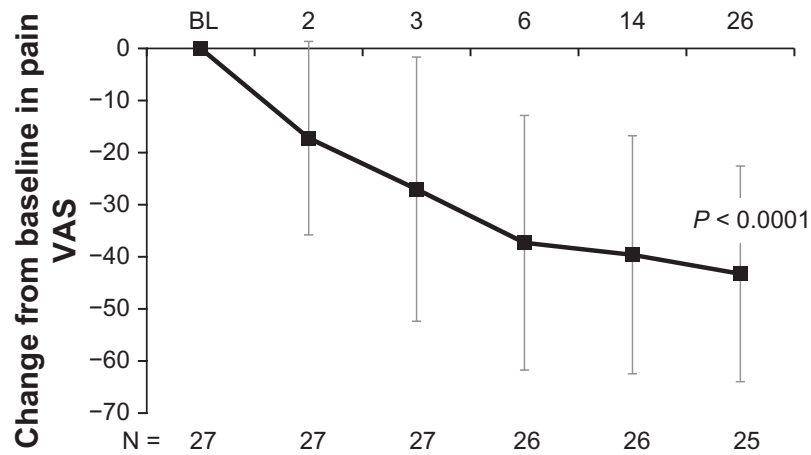

B

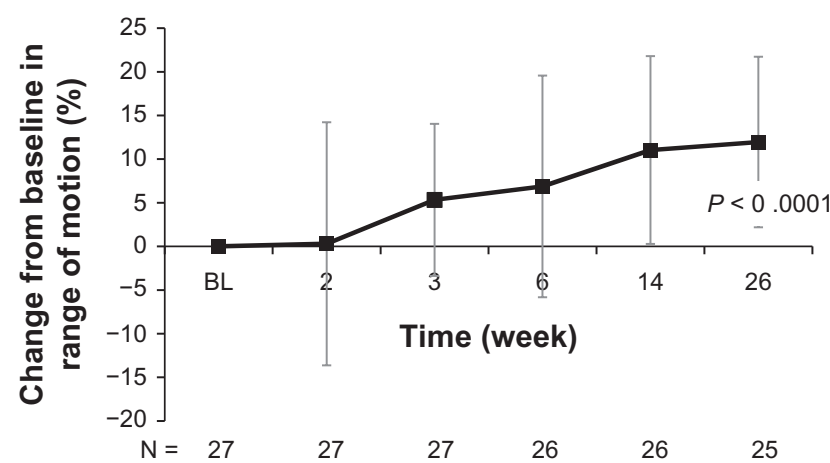

Figure I Intra-articular injection of high molecular weight hyaluronate significantly improved pain and range of motion at 26 weeks of follow-up for study subjects with glenohumeral osteoarthritis. Subjects were treated with one injection per week for 3 weeks of high molecular weight hyaluronate and followed up after treatment for 26 weeks. Data represents mean change from baseline (week I) \pm standard deviation after treatment with high molecular weight hyaluronate in (A) pain visual analog scores and (B)\% range of motion over 26 weeks of follow-up.

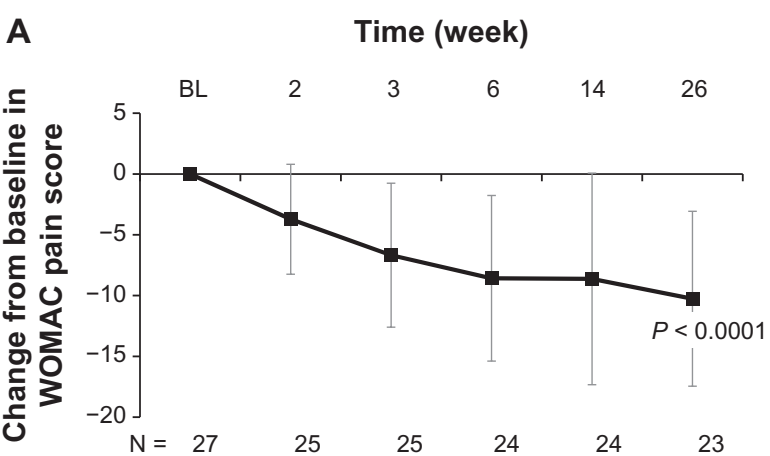

B Time (week)

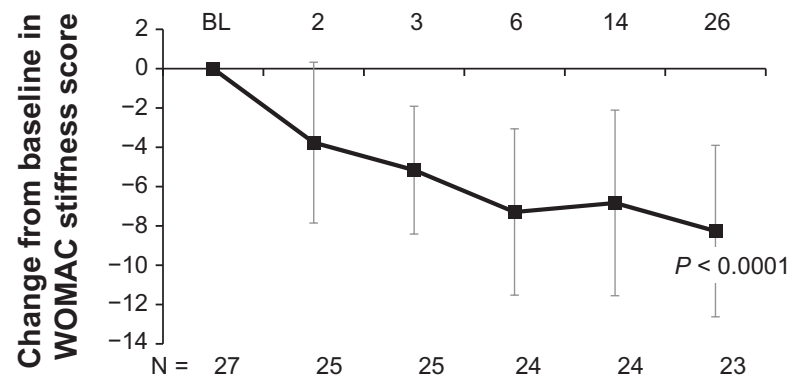

C Time (week)

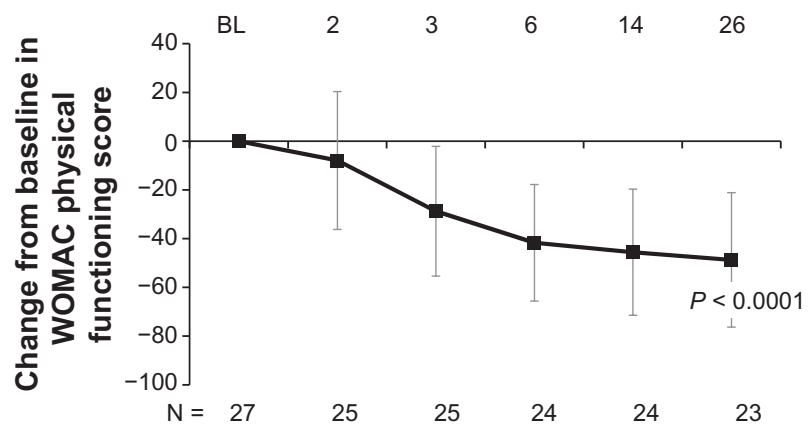

Figure 2 Intra-articular injection of high molecular weight hyaluronate significantly improved WOMAC pain, stiffness, and physical functioning at 26 weeks of follow-up for study subjects with glenohumeral osteoarthritis. Subjects were treated with one injection per week for 3 weeks of high molecular weight hyaluronate and followed-up after treatment for 26 weeks. Data represent mean change from baseline (week I) \pm standard deviation after treatment with high molecular weight hyaluronate over 26 weeks of follow-up in (A) WOMAC pain scores, (B) WOMAC stiffness scores, and (C) WOMAC physical functioning scores.

Abbreviation: WOMAC, Western Ontario and McMaster Universities Arthritis Index.

and six subjects (22.2\%) were considered to be nonresponders (Figure 5). Logistic regression examined the impact of age and gender on the likelihood of being a responder, and no significant effect of either of these variables was found.

\section{Safety}

Fourteen subjects reported a total of 47 adverse events. The most common adverse event was shoulder pain (nine reports); all but two adverse events were mild or moderate in severity (the severe adverse events were lethargy and slurred speech). 


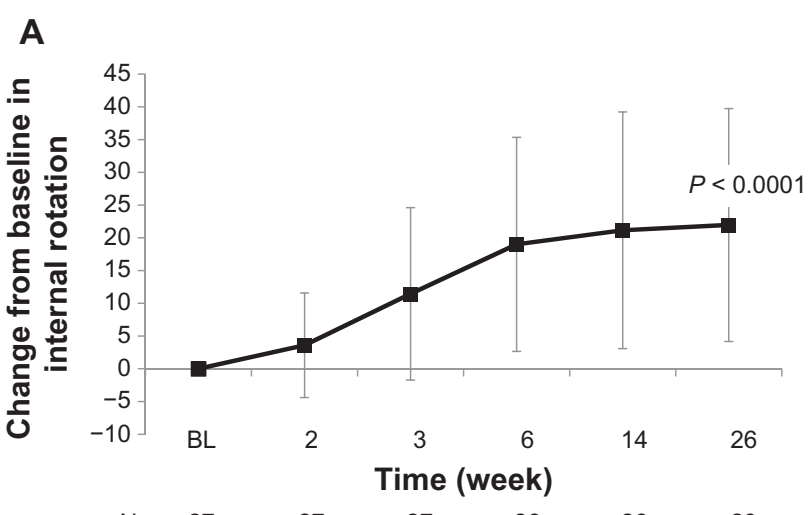

B
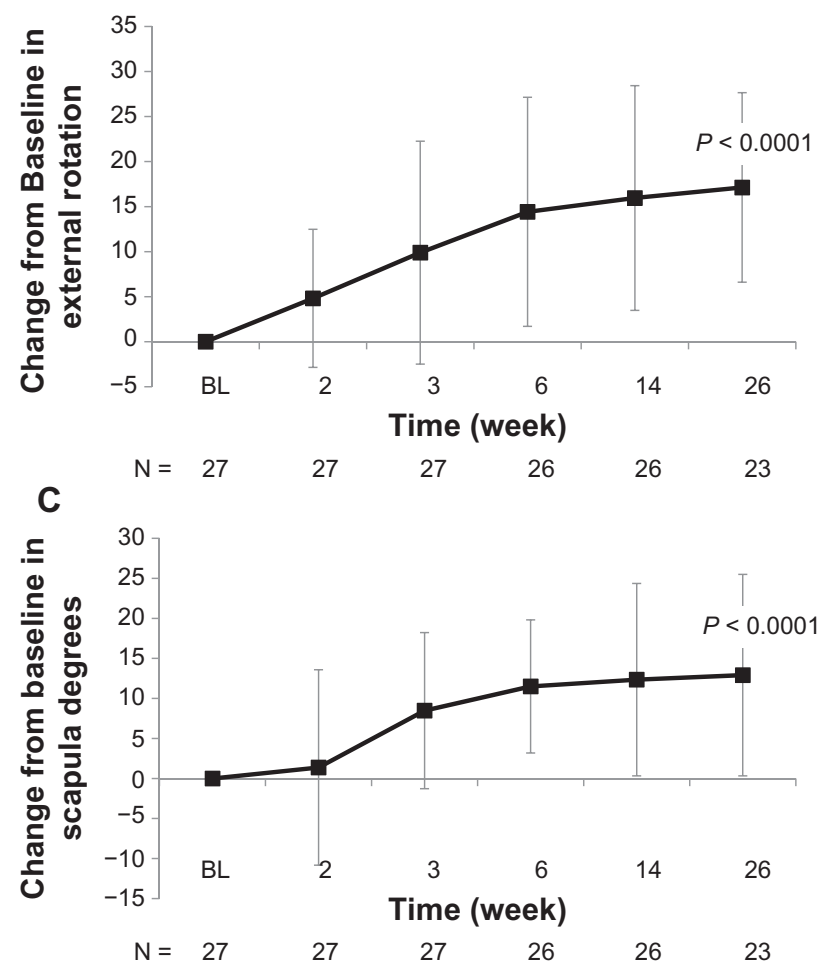

Figure 3 Intra-articular injection of high molecular weight hyaluronate significantly improved internal and external rotation and scapula degrees at 26 weeks of follow-up for study subjects with glenohumeral osteoarthritis. Subjects were treated with one injection per week for 3 weeks of high molecular weight hyaluronate and followed up after treatment for 26 weeks. Data represent mean change from baseline (week I) \pm standard deviation after treatment with high molecular weight hyaluronate over 26 weeks of follow-up in (A) internal shoulder rotation, (B) external shoulder rotation, and $(\mathbf{C})$ scapula degrees.

There were no serious adverse events, and no adverse events were considered to be related to treatment. No subjects discontinued treatment due to adverse events.

\section{Discussion}

Results from this exploratory, small-scale, open-label study showed that treatment of chronic shoulder pain secondary to glenohumeral osteoarthritis with high molecular weight hyaluronate decreased pain and increased range of motion.
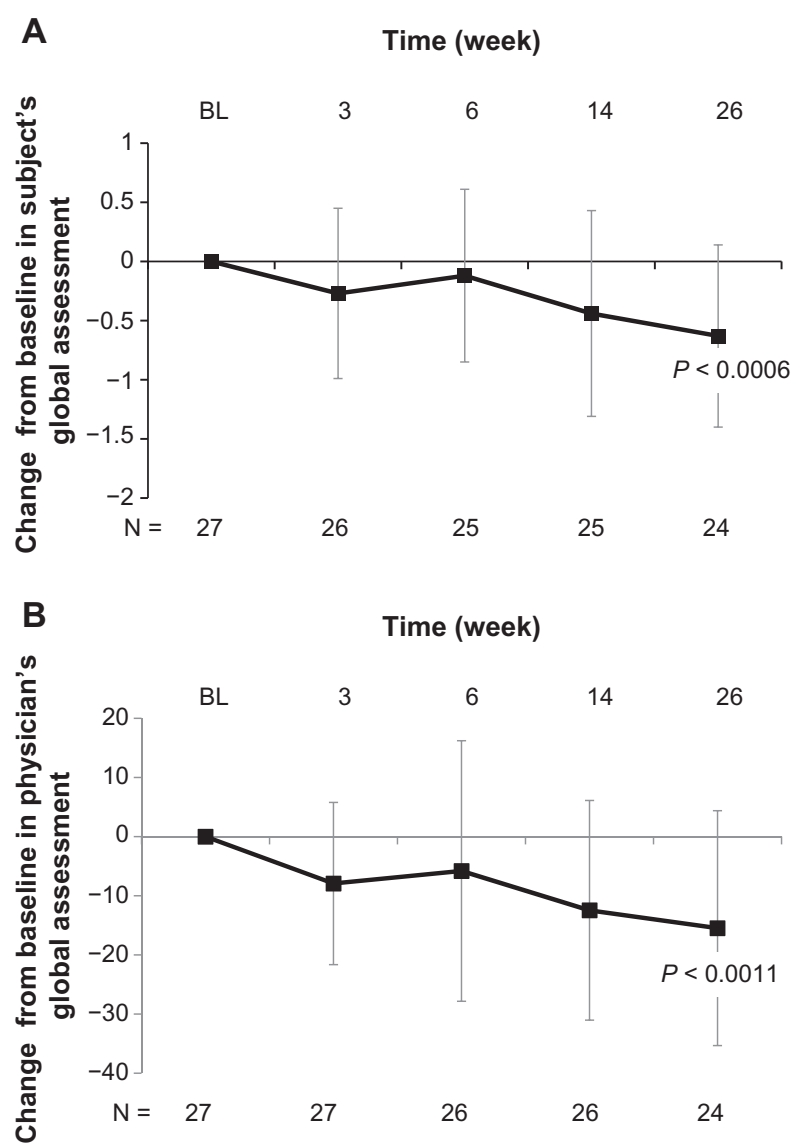

Figure $\mathbf{4}$ Intra-articular injection of high molecular weight hyaluronate significantly improved subject's and physician's global assessments at 26 weeks of follow-up for study subjects with glenohumeral osteoarthritis. Subjects were treated with three weekly injections of high molecular weight hyaluronate and followed up after treatment for 26 weeks. Data represent mean change from baseline (week I) \pm standard deviation after treatment with high molecular weight hyaluronate over 26 weeks of follow-up in (A) subject's global assessments and (B) physician's global assessments.

Treatment with intra-articular high molecular weight hyaluronate also improved WOMAC pain, stiffness, and physical function, as well as subject's and physician's global assessments. In addition, $77.8 \%$ of subjects were considered high responders to treatment based on OMERACT-OARSI Proposition D criteria. Treatment with high molecular weight hyaluronate had an acceptable safety profile in subjects with chronic shoulder pain secondary to osteoarthritis. No treatment-related adverse events were reported, and all-causality adverse events were almost all mild or moderate in severity.

The results of this study are consistent with other reports indicating that intra-articular hyaluronate is effective for the treatment of persistent shoulder pain associated with glenohumeral osteoarthritis. ${ }^{11-13}$ These results also support the view expressed by the American Academy of Orthopedic Surgeons that the use of injectable viscosupplementation is an option for treating patients with glenohumeral joint osteoarthritis, and that hyaluronate injections have significant benefit 


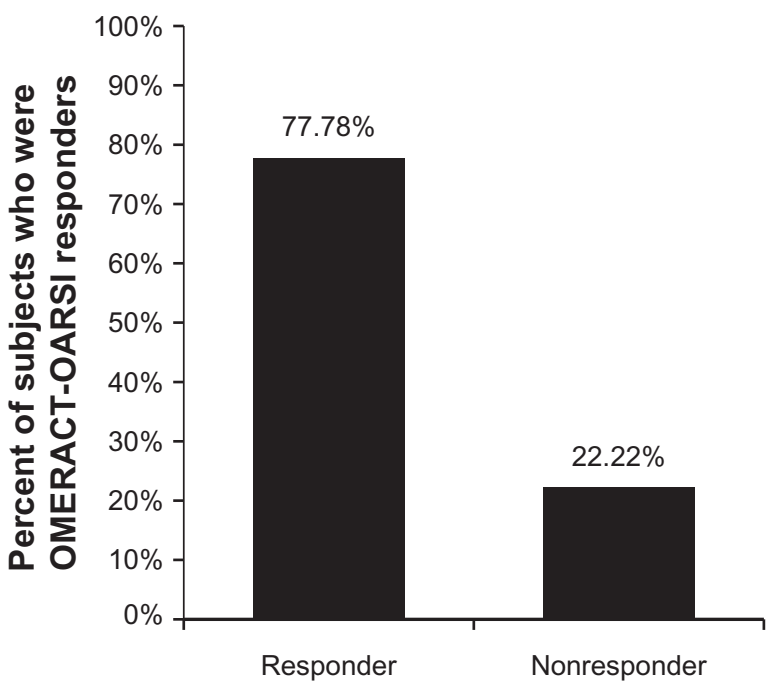

Figure 5 Based on Outcome Measures in Rheumatoid Arthritis Clinical TrialsOsteoarthritis Research Society International Proposition D criteria, 77.78\% of study subjects treated with intra-articular high molecular weight hyaluronate were considered high responders. Subjects were treated with three weekly injections of high molecular weight hyaluronate and followed up after treatment for 26 weeks. Twenty-one patients $\mathbf{( 7 7 . 7 8 \% )}$ ) were considered high responders, while the remaining six patients were considered nonresponders.

in pain relief, range of motion, and quality of life. ${ }^{5}$ The safety of high molecular weight hyaluronate in the present study is consistent with results from two larger scale trials of this preparation in patients with knee osteoarthritis. ${ }^{17,18}$

Although nonavian-derived high molecular weight hyaluronate was not directly compared with hyaluronates derived from avian sources in this study, bioengineered, nonavian, and nonchemically cross-linked high molecular weight hyaluronate may have safety advantages over avian-derived hyaluronate preparations as well as hyaluronate preparations that achieve high molecular weight using chemically induced cross-linking. No adverse effects of avian-derived hyaluronate were reported in one small-scale, double-blind, placebo-controlled study for hyaluronate treatment of rotator cuff lesions without complete tears. ${ }^{15}$ However, hyaluronan preparations that achieve high molecular weight via chemically induced cross-linking have been associated with pseudosepsis, a rare localized inflammatory reaction characterized by joint effusion, pain, and infiltration of inflammatory cells, in several large-scale studies for hyaluronate treatment of knee osteoarthritis. ${ }^{19-28}$

The major limitations of this study are that it is a smallscale, open-label study which did not include a placebo control group. However, the results of this and other studies suggest that there may be a need to evaluate further the safety and efficacy of intra-articular hyaluronate for treatment of chronic shoulder pain in a large-scale, multicenter, doubleblind, placebo-controlled clinical trial.
A recent review of treatments for chronic shoulder pain concluded that "... there are no known interventions that alter the natural history of early osteoarthritis; therefore, the main objectives in treatment are to control pain and restore function". ${ }^{25}$ There is a clear need for treatment with the potential to alter the natural history of shoulder osteoarthritis. While there is no evidence regarding disease-modifying activity for intra-articular hyaluronate in patients with shoulder osteoarthritis, a growing body of evidence supports the possibility that this treatment may have disease-modifying activity in knee osteoarthritis, including effects in the synovium and extracellular matrix of the articular cartilage, subchondral bone, matrix proteoglycans, and collagens. ${ }^{19,28}$ Further study will be necessary to address the potential disease-modifying properties of viscosupplementation agents.

\section{Conclusion}

The results of this study and others suggest that intra-articular high molecular weight hyaluronate may be a safe and effective treatment for shoulder pain associated with glenohumeral osteoarthritis. However, this was a small-scale, open-label study which did not include a placebo control group. A largescale, multicenter, double-blind, placebo-controlled clinical trial is necessary to evaluate further the safety and efficacy of intra-articular hyaluronate for treatment of chronic shoulder pain. In addition, although a growing body of evidence supports the possibility that intra-articular hyaluronates may have disease-modifying activity in knee osteoarthritis, there is no evidence regarding disease-modifying activity for intra-articular hyaluronate in patients with shoulder osteoarthritis.

\section{Acknowledgments}

The author is indebted to all patients who participated in this study. Data analysis was done by Hind T Hatoum and Company, Chicago, IL.

\section{Disclosure}

The study was supported by Ferring Pharmaceuticals Inc.

\section{References}

1. Pope DP, Croft PR, Pritchard CM, Silman AJ. Prevalence of shoulder pain in the community: the influence of case definition. Ann Rheum Dis. 1997;56(5):308-312.

2. van der Heijden GJ. Shoulder disorders: A state-of-the-art review. Baillieres Best Pract Res Clin Rheumatol. 1999;13(2):287-309.

3. Andrews JR. Diagnosis and treatment of chronic painful shoulder: review of nonsurgical interventions. Arthroscopy. 2005;21(3):333-347.

4. Wofford JL, Mansfield RJ, Watkins RS. Patient characteristics and clinical management of patients with shoulder pain in U.S. primary care settings: secondary data analysis of the National Ambulatory Medical Care Survey. BMC Musculoskelet Disord. 2005;6:4. 
5. Izquierdo R, Voloshin I, Edwards S, et al. Treatment of glenohumeral osteoarthritis. J Am Acad Orthop Surg. 2010;18(6):375-382.

6. Sandor R. Adhesive capsulitis: optimal treatment of "frozen shoulder". Available from: https://physsportsmed.org/doi/10.3810/ psm.2000.09.1200. Accessed June 6, 2011.

7. Burbank KM, Stevenson JH, Czarnecki GR, Dorfman J. Chronic shoulder pain: Part II. Treatment. Am Fam Physician. 2008;77(4):493-497.

8. Moskowitz RW, Blaine TA. An overview of treatment options for persistent shoulder pain. Am J Orthop (Belle Mead NJ). 2005; 34(12 Suppl):10-15.

9. Croft P, Pope D, Silman A. The clinical course of shoulder pain: prospective cohort study in primary care. Primary Care Rheumatology Society Shoulder Study Group. BMJ. 1996;313(7057):601-602.

10. van der Windt DA, Koes BW, Boeke AJ, Deville W, De Jong BA, Bouter LM. Shoulder disorders in general practice: prognostic indicators of outcome. Br J Gen Pract. 1996;46(410):519-523.

11. Saito S, Furuya T, Kotake S. Therapeutic effects of hyaluronate injections in patients with chronic painful shoulder: a meta-analysis of randomized controlled trials. Arthritis Care Res (Hoboken). 2010;62(7):1009-1018.

12. Silverstein E, Leger R, Shea KP. The use of intra-articular hylan G-F 20 in the treatment of symptomatic osteoarthritis of the shoulder: a preliminary study. Am J Sports Med. 2007;35(6):979-985.

13. Blaine T, Moskowitz R, Udell J, et al. Treatment of persistent shoulder pain with sodium hyaluronate: A randomized, controlled trial. a multicenter study. J Bone Joint Surg Am. 2008;90(5):970-979.

14. Tagliafico A, Serafini G, Sconfienza LM, et al. Ultrasound-guided viscosupplementation of subacromial space in elderly patients with cuff tear arthropathy using a high weight hyaluronic acid: prospective open-label non-randomized trial. Eur Radiol. 2011;21(1):182-187.

15. Chou WY, Ko JY, Wang FS, et al. Effect of sodium hyaluronate treatment on rotator cuff lesions without complete tears: a randomized, double-blind, placebo-controlled study. J Shoulder Elbow Surg. 2010;19(4):557-563.

16. Euflexxa. United States prescribing information. 2009. Available from: http://www.euflexxa.com/files/euflexxa_physician.pdf. Accessed July 15, 2011.
17. Altman RD, Rosen JE, Bloch DA, Hatoum HT, Korner P. A doubleblind, randomized, saline-controlled study of the efficacy and safety of EUFLEXXA for treatment of painful osteoarthritis of the knee, with an open-label safety extension (the FLEXX trial). Semin Arthritis Rheum. 2009;39(1):1-9.

18. Kirchner M, Marshall D. A double-blind randomized controlled trial comparing alternate forms of high molecular weight hyaluronan for the treatment of osteoarthritis of the knee. Osteoarthritis Cartilage. 2006;14(2):154-162.

19. Goldberg VM, Buckwalter JA. Hyaluronans in the treatment of osteoarthritis of the knee: Evidence for disease-modifying activity. Osteoarthritis Cartilage. 2005;13(3):216-224.

20. Goldberg VM, Coutts RD. Pseudoseptic reactions to hylan viscosupplementation: diagnosis and treatment. Clin Orthop Relat Res. 2004;419:130-137.

21. Goomer RS, Leslie K, Maris T, Amiel D. Native hyaluronan produces less hypersensitivity than cross-linked hyaluronan. Clin Orthop Relat Res. 2005;434:239-245.

22. Leopold SS, Warme WJ, Pettis PD, Shott S. Increased frequency of acute local reaction to intra-articular hylan GF-20 (Synvisc) in patients receiving more than one course of treatment. J Bone Joint Surg Am. 2002;84-A(9):1619-1623.

23. Marino AA, Waddell DD, Kolomytkin OV, Pruett S, Sadasivan KK, Albright JA. Assessment of immunologic mechanisms for flare reactions to Synvisc. Clin Orthop Relat Res. 2006;442:187-194.

24. Martens PB. Bilateral symmetric inflammatory reaction to hylan G-F 20 injection. Arthritis Rheum. 2001;44(4):978-979.

25. Millett PJ, Gobezie R, Boykin RE. Shoulder osteoarthritis: diagnosis and management. Am Fam Physician. 2008;78(5):605-611.

26. Morton AH, Shannon P. Increased frequency of acute local reaction to intra-articular hylan G-F 20 (Synvisc) in patients receiving more than one course of treatment. J Bone Joint Surg Am. 2003;85-A(10):2050.

27. Rees JD, Wojtulewski JA. Systemic reaction to viscosupplementation for knee osteoarthritis. Rheumatology (Oxford). 2001;40(12):1425-1426.

28. Yatabe T, Mochizuki S, Takizawa M, et al. Hyaluronan inhibits expression of ADAMTS4 (aggrecanase-1) in human osteoarthritic chondrocytes. Ann Rheum Dis. 2009;68(6):1051-1058.
Medical Devices: Evidence and Research

\section{Publish your work in this journal}

Medical Devices: Evidence and Research is an international, peerreviewed, open access journal that focuses on the evidence, technology, research, and expert opinion supporting the use and application of medical devices in the diagnosis, treatment and management of clinical conditions and physiological processes. The identification of novel

\section{Dovepress}

devices and optimal use of existing devices which will lead to improved clinical outcomes and more effective patient management and safety is a key feature. The manuscript management system is completely online and includes a quick and fair peer-review system. Visit http://www. dovepress.com/testimonials.php to read real quotes from authors. 